\title{
AN APPLICATION OF A THEOREM OF HUBER IN HOLOMORPHIC FOLIATION THEORY
}

\author{
HANS-JÖRG REIFFEN \\ FB Mathematik/Informatik, Albrechtstr. 28, D-49076 Osnabrück, Germany
}

Introduction. In the following we are concerned with 1-codimensional holomorphic foliations on a connected paracompact complex manifold $X$ of dimension $n$.

Let $U$ be an open subset of $X$ and $f: U \rightarrow C$ a holomorphic submersion onto a 1-dimensional complex manifold $C . f: U \rightarrow C$ is called a regular local holomorphic foliation of codimension one. Two regular local holomorphic foliations $f_{1}: U_{1} \rightarrow$ $C_{1}, f_{2}: U_{2} \rightarrow C_{2}$ are called compatible, if for every $x \in U_{1} \cap U_{2}$ there exist an open neighborhood $W \subset U_{1} \cap U_{2}$ of $x$ and a biholomorphic mapping $g: f_{1}(W) \rightarrow$ $f_{2}(W)$ such that $f_{2}=g \circ f_{1}$ on $W$. A (global) regular holomorphic foliation $\mathcal{F}$ of codimension one on $X$ is a system $\left\{f_{j}: U_{j} \rightarrow C_{j}: j \in J\right\}$ of compatible regular local holomorphic foliations of codimension one such that $\bigcup_{j \in J} U_{j}=X$.

We identify two regular foliations $\mathcal{F}_{1}, \mathcal{F}_{2}$ on $X$ if every local foliation of $\mathcal{F}_{1}$ is compatible with every local foliation of $\mathcal{F}_{2}$. In the following we assume that every regular foliation $\mathcal{F}$ on $X$ contains every local foliation which is compatible with those of $\mathcal{F}$. By a theorem of Frobenius there is a one to one correspondence between the system of regular holomorphic foliations $\mathcal{F}$ of codimension 1 on $X$ and the system of subsheaves $\Omega^{\prime}$ of the sheaf $\Omega^{1}$ of holomorphic Pfaffian forms on $X$ such that $\Omega^{1} / \Omega^{\prime}$ is a locally free $\mathcal{O}$-sheaf of rank $n-1$ and $\omega \wedge d \omega=0$ for every $\omega \in \Omega_{x}^{\prime}, x \in X$.

Let $\mathcal{F}$ be a regular holomorphic foliation on $X$ of codimension 1 . A subset $L$ of $X$ is called a local leaf or a plaque of $\mathcal{F}$, if there is a local holomorphic foliation $f: U \rightarrow C$ of $\mathcal{F}$ such that $L$ is a connected component of a fiber of $f$. The relatively open subsets of the local leaves of $\mathcal{F}$ constitute a base of a topology $\mathcal{T}$ on $X$. $\mathcal{T}$ is called the $\mathcal{F}$-topology. $(X, \mathcal{T})$ is a complex manifold of dimension $n-1$. It is not connected. The connected components $L$ of $(X, \mathcal{T})$ are called

1991 Mathematics Subject Classification: 32L30, 32H20.

The paper is in final form and no version of it will be published elsewhere. 
leaves of $\mathcal{F}$. We denote by $X / \mathcal{F}$ the space of all leaves and by $\pi: X \rightarrow X / \mathcal{F}$ the natural projection We equip $X / \mathcal{F}$ with the quotient topology and the natural ringed structure. Then $\pi$ is an open morphism. In [Ho 1] Holmann has proved the following leaf-space theorem.

THEOREM H. $X / \mathcal{F}$ is a complex space if and only if it is Hausdorff.

If $X / \mathcal{F}$ is a complex space then it is a Riemannian surface. Theorem $H$ is also true under more general conditions, especially for foliations of higher codimension.

We are concerned with (singular) 1-codimensional holomorphic foliations on $X$. Those are pairs $\left(\mathcal{F}^{\prime}, A^{\prime}\right)$, in which $A^{\prime} \subset X$ is an analytic subset of codimension $\geq 2$ and $\mathcal{F}^{\prime}$ is a regular holomorphic foliation of codimension 1 on $X \backslash A^{\prime}$. We identify two singular foliations $\left(\mathcal{F}_{1}^{\prime}, A_{1}^{\prime}\right),\left(\mathcal{F}_{2}^{\prime}, A_{2}^{\prime}\right)$ if $\mathcal{F}_{1}^{\prime}=\mathcal{F}_{2}^{\prime}$ on $X \backslash\left(A_{1}^{\prime} \cup A_{2}^{\prime}\right)$. If $A$ is the smallest possible exceptional analytic subset of a singular foliation $\mathcal{F}=\left(\mathcal{F}^{*}, A\right)$, we call sing $\mathcal{F}=A$ the singular locus of $\mathcal{F} . \mathcal{F}^{*}$ is the maximal corresponding regular foliation of $\mathcal{F}$. It is a foliation on $X^{*}=X \backslash \operatorname{sing} \mathcal{F}$. There is a one to one correspondence between the system of holomorphic foliations $\mathcal{F}$ of codimension 1 on $X$ and the system of coherent analytic subsheaves $\Omega^{\prime}$ of $\Omega^{1}$ such that $\Omega^{1} / \Omega^{\prime}$ is a $\mathcal{O}$-sheaf without torsion of rank $n-1$ and $\omega \wedge d \omega=0$ for every $\omega \in \Omega_{x}^{\prime}, x \in X$. We get $\operatorname{sing} \mathcal{F}=\operatorname{sing} \Omega^{1} / \Omega^{\prime}$, where sing $\Omega^{1} / \Omega^{\prime}$ is the set of all points $x \in X$ such that $\Omega_{x}^{1} / \Omega_{x}^{\prime}$ is not free. For the general theory of singular holomorphic foliations compare $[\mathrm{B} / \mathrm{R}]$.

Let $\mathcal{F}$ be a holomorphic foliation on $X$ of codimension 1. Let $U$ be an open subset of $X$ and $f: U \rightarrow C$ an open holomorphic mapping onto a 1-dimensional complex manifold $C$. $f$ is called an integral of $\mathcal{F}$ on $U$ if $f$ is locally constant on $U^{*}=U \cap X^{*}$ relating to the leaf topology of $\mathcal{F}^{*}$, i.e. if $f$ defines a regular local foliation belonging to $\mathcal{F}^{*}$ on a dense open subset of $U^{*}$. If theorem $H$ is true the projection $\pi: X \rightarrow X / \mathcal{F}$ is an integral of $\mathcal{F}$. The notion of an integral is a generalization of the notion of a local foliation. For the general theory of integrals compare $[\mathrm{B} / \mathrm{R}]$ and $[\mathrm{Rf} 2]$. In $[\mathrm{M} / \mathrm{M}]$ Mattei and Moussu gave a topological description of integrability in codimension 1.

Theorem M/M. Let $a \in X$. The following statements are equivalent:

(1) There exist an open neighborhood $U$ of a and an integral of $\mathcal{F}$ on $U$.

(2) There exists an open neighborhood $V$ of a such that

(a) every leaf of $\mathcal{F} \mid V^{*}$ is a closed subset of $V^{*}$ and

(b) a is cluster point of a countable number of leaves of $\mathcal{F} \mid V^{*}$ at the most.

Obviously (2) is necessary for (1). Even more, from (1) we conclude a stronger version of (b), namely

$\left(\mathrm{b}^{*}\right)$ a is cluster point of a finite number of leaves of $\mathcal{F} \mid V^{*}$.

Corresponding to the conditions (2) of theorem $\mathrm{M} / \mathrm{M}$ we define

0.1. Definition. $\mathcal{F}$ is called geometrically simple if the following conditions are satisfied: 
(a) every leaf of $\mathcal{F}^{*}$ is a closed subset of $X^{*}$ and

(b) every point $x \in \operatorname{sing} \mathcal{F}$ is a cluster point of a countable number of leaves of $\mathcal{F}^{*}$ at the most.

Because of theorem $\mathrm{M} / \mathrm{M}$ we may replace (b) by a stronger condition like ( $\left.\mathrm{b}^{*}\right)$.

In section 2 we prove the following theorem.

0.2. Theorem. Let $\mathcal{F}$ be geometrically simple and $X$ compact. Then there exists an integral of $\mathcal{F}$ on $X$.

The theorem is a corollary of the following technical theorem.

0.3. Theorem. Let $\Gamma$ be a set of leaves of $\mathcal{F}^{*}$. Assume that

(a) every $L \in \Gamma$ is a closed subset of $X^{*}$,

(b) $\Gamma$ is a locally finite family of subsets of $X^{*}$,

(c) for every $L \in \Gamma$ the space

$$
\left(X^{*} / \mathcal{F}^{*}\right) \backslash\{M \in \Gamma: M \neq L\}
$$

is Hausdorff,

(d) for every $x \in \operatorname{sing} \mathcal{F}$ there exists at least one leaf $L$ of $\mathcal{F}^{*}, L \notin \Gamma$, such that $x \notin \bar{L}$.

Then there exists an integral of $\mathcal{F}$ on $X$.

From condition (c) in 0.3 we get integrals by theorem $H$. In section 1 we will combine technics to glue them together. For that purpose we use methods of hyperbolic analysis.

We call $\mathcal{F}$ locally integrable, if for every $a \in X$ there exists an integral $f$ of $\mathcal{F}$ on an open neighborhood of $a$. By the aid of theorem M/M we get:

$\mathcal{F}$ is locally integrable iff $\mathcal{F}$ is locally geometrically simple.

Let $\mathcal{F}$ be locally integrable then we can define local leaves, $\mathcal{F}$-topology and global leaves in a similar way as in the regular case (comp. [B/R], [Rf 2]). Therefore we can also define the leaf-space $X / \mathcal{F}$ and the projection $\pi: X \rightarrow X / \mathcal{F} . X / \mathcal{F}$ is a ringed space in a natural way and $\pi$ a morphism.

Using the result of [Rf 1] one can show that $\pi$ is open.

An integral $f: U \rightarrow C$ of $\mathcal{F}$ is called simple if all fibers of $f$ are connected. We conclude:

Let $\mathcal{F}$ be locally integrable. Then the following statements are equivalent:

(1) There exists a simple integral $f: X \rightarrow C$ of $\mathcal{F}$.

(2) $X / \mathcal{F}$ is a complex space.

In this case we can identify $C=X / \mathcal{F}, f=\pi$ and, especially $X / \mathcal{F}$ is a Riemannian surface.

We show in the situation of theorem 0.2 that there exists a simple integral of $\mathcal{F}$ on $X$. Therefore we get: 
0.4. Corollary. Let $\mathcal{F}$ be geometrically simple and $X$ compact. Then $X / \mathcal{F}$ is a Riemannian surface.

By the way we also show in the situation of theorem 0.3 that there exists an integral of a special type.

Let $\mathcal{F}$ be geometrically simple. We denote by $\operatorname{sh} \mathcal{F}$ the $\mathcal{F}$-saturated hull of $\operatorname{sing} \mathcal{F}$, i.e. the union of all leaves of $\mathcal{F}$ cutting sing $\mathcal{F}$. The following corollary of 0.3 is a generalization of theorem $H$ in codimension 1 .

0.5. Corollary. Assume that $\mathcal{F}$ is geometrically simple and sh $\mathcal{F}$ is an analytic subset of $X$. Then $X / \mathcal{F}$ is a Riemannian surface if and only if it is Hausdorff.

In the last section we will make some remarks on the proof of theorem $\mathrm{M} / \mathrm{M}$ and generalizations of theorem $\mathrm{M} / \mathrm{M}$, theorem 0.2 and corollary 0.4 .

1. Some extension theorems. In this section let $X, X_{1}, X_{2}$ be arbitrary complex manifolds and $C, C_{1}, C_{2}$ Riemannian surfaces. By $\mathcal{O}\left(X_{1}, X_{2}\right)$ we denote the set of all holomorphic mappings $f: X_{1} \rightarrow X_{2}$. Let be $D=\{t \in \mathbb{C}:|t|<1\}$ and $D^{*}:=D \backslash\{0\}$. By $\tilde{C}$ we denote the universal covering of $C$. It is well known that $\tilde{C}$ is isomorphic to $D, \mathbb{C}$ or $\mathbb{P}^{1}$ (comp. for example [Fo]). By removing at most three points we get a Riemannian surface $C^{\prime}$ from $C$ such that $\tilde{C}^{\prime} \cong D$.

It is well known that for a Riemannian surface $C$ the modern notion of hyperbolicity coincides with the classical one, i.e. $C$ is hyperbolic iff $\tilde{C} \cong D$ (comp. $[\mathrm{Ko}])$.

In $[\mathrm{Hu}]$ Huber has proved the following extension theorem.

Huber Theorem. Let $C$ be hyperbolic and $f \in \mathcal{O}\left(D^{*}, C\right)$. If there exists a sequence $z_{\nu}$ in $D^{*}$ such that $\lim z_{\nu}=0$ and $\lim f\left(z_{\nu}\right)$ exists then $f$ has an extension $\tilde{f} \in \mathcal{O}(D, C)$.

Huber proved his theorem using the Kobayshi-Royden length of curves in hyperbolic Riemannian surfaces. In $[\mathrm{Kw}]$ Kwack generalized the theorem by replacing $C$ by an arbitrary hyperbolic manifold $X$.

With the aid of Huber's theorem we get:

1.1. Lemma. Let $c_{j} \in C_{j}, j=1,2$, and $\varphi \in \mathcal{O}\left(C_{1} \backslash\left\{c_{1}\right\}, C_{2} \backslash\left\{c_{2}\right\}\right)$ injective. If there exists a sequence $x_{\nu}$ in $C_{1} \backslash\left\{c_{1}\right\}$ such that $\lim x_{\nu}=c_{1}$ and $\lim f\left(x_{\nu}\right)=c_{2}$ then $\varphi$ has an extension $\tilde{\varphi} \in \mathcal{O}\left(C_{1}, C_{2}\right)$.

Proof. We may assume that $C_{2}$ is hyperbolic. Otherwise we remove some points of $C_{2} \backslash\left\{c_{2}\right\}$. Now apply Huber's theorem.

We need also the following lemma:

1.2. Lemma. Let $A$ be an analytic subset of $X$ of codimension $\geq 2$ and $f \in$ $\mathcal{O}(X \backslash A, C)$. Assume that for every $a \in A$ there exists a point $c \in C$ such that $a$ is no cluster point of $f^{-1}(c)$. Then there exists a holomorphic extension $\tilde{f} \in \mathcal{O}(X, C)$ of $f$. 
Proof. We must extend $f$ locally. Therefore we may assume the following:

$$
X=D^{n}, \quad A=D^{m}, \quad m \leq n-2,
$$

there exists a point $c \in C \backslash f(X \backslash A)$.

We have $\tilde{C} \cong D$ or $\tilde{C} \cong \mathbb{C}$ or $C \cong \mathbb{P}^{1}$. If $C \cong \mathbb{P}^{1}$ we remove $c$. Therefore we may assume that $\tilde{C} \cong D$ or $\tilde{C} \cong \mathbb{C}$.

Because $X \backslash A$ is simply connected we get a mapping $g \in \mathcal{O}(X \backslash A, \tilde{C})$ such that $f=\pi \circ g, \pi$ being the projection $\pi: \tilde{C} \rightarrow C$. We extend $g$ by the classical Riemannian extension theorem and set $\tilde{f}=\tilde{g} o \pi, \tilde{g}$ being the extension of $g$.

2. The proofs of $\mathbf{0 . 2}, \mathbf{0 . 3}$ and $\mathbf{0 . 5}$. In this section let $X$ be a connected paracompact complex manifold of dimension $n$ and $\mathcal{F}$ a holomorphic foliation on $X$ of codimension 1 .

2.1. Definition. Let $\mathcal{F}$ be locally integrable. Two leaves $L, L^{\prime}$ of $\mathcal{F}$ are called not separable, $L \leftrightarrow L^{\prime}$, if $U \cap U^{\prime} \neq \emptyset$ for every neighborhood $U$ resp. $U^{\prime}$ of $L$ resp. $L^{\prime}$ in $X / \mathcal{F}$.

2.2. Remark and Definition. Let $f: X \rightarrow C$ be an integral of $\mathcal{F}$. Then there exists a unique mapping $\tilde{f}: X / \mathcal{F} \rightarrow C$ such that $\tilde{f}$ o $\pi=f$. $\tilde{f}$ is a surjective open morphism. We get: $L \leftrightarrow M \Rightarrow \tilde{f}(L)=\tilde{f}(M)$. $f$ is called maximally separating (m.s.) if $\tilde{f}(L)=\tilde{f}(M) \Leftrightarrow L \leftrightarrow L^{\prime}$.

Let $f: X \rightarrow C$ be a m.s. integral. Then we can identify $C$ with the quotient $(\widetilde{X / \mathcal{F}})$ of $(X / \mathcal{F})$ by $\leftrightarrow$ and $f$ with the projection $\tilde{\pi}: X \rightarrow(\widetilde{X / \mathcal{F}})$.

A simple integral is a m.s. integral.

Pro of of 0.3 . In 0.3 we allow that $\Gamma=\emptyset$. Then we only have the condition, that $X^{*} / \mathcal{F}^{*}$ is Hausdorff, and condition (d).

First assume that $\sin g \mathcal{F}=\emptyset$, i.e. that $\mathcal{F}$ is a regular foliation. If $\Gamma=\emptyset$ then 0.3 follows by theorem $H$. Let $\Gamma \neq \emptyset$.

Let $L \in \Gamma$. Then $L$ is a closed subset of $X$. By a theorem of Holman (comp. [Ho 1]) we get that $L$ is an analytic subset of $X$. Because $\Gamma$ is locally finite we conclude that $A=\bigcup_{L \in \Gamma} L$ is an analytic subset of $X$. The sets $X_{0}=X \backslash A$ and $X_{L}=X_{0} \cup L, L \in \Gamma$ are open subsets of $X$. By theorem $H$ we can conclude that

$$
C_{0}=(X / \mathcal{F}) \backslash \Gamma \quad \text { and } \quad C_{L}=C_{0} \cup\{L\}, L \in \Gamma,
$$

are Riemannian surfaces. The natural projections $f_{0}=X_{0} \rightarrow C_{0}, f_{L}: X_{L} \rightarrow C_{L}$ are simple and therefore m.s. integrals. Now we consider the system $\mathcal{I}$ of all m.s. integrals $f: U \rightarrow C$, in which $U$ is an open $\mathcal{F}$-saturated subset of $X$ containing $X_{0} . f_{0}=X_{0} \rightarrow C_{0}$ and $f_{L}: X_{L} \rightarrow C_{L}$ are elements of $\mathcal{I}$. If we norm the m.s. integrals as described following 2.2 then we can identify every element $f: U \rightarrow C$ of $\mathcal{I}$ with $U$. If $f: U \rightarrow C, f^{\prime}: U^{\prime} \rightarrow C^{\prime}$ belong to $\mathcal{I}, U \subset U^{\prime}$, then we get $C \subset C^{\prime}$ in a natural way. Therefore the inclusion of the domains of definiton of the integrals gives an ordering $\leq$ on $\mathcal{I}$. The condition of Zorn's lemma is satisfied. We consider a maximal element $f: U \rightarrow C$ of $\mathcal{I}$. 
By an indirect argument we show that $U=X$. Assume that there exists an element $L \in \Gamma, L \not \subset U$. We have $C_{0}=C \cap C_{L}$ in a natural way. Let $S=f(U \cap A)$. There exist two alternatives.

1st case: For every $\sigma \in S$ there exist neighborhoods $W$ of $\sigma$ in $C$ and $V$ of $L$ in $C_{L}$ such that $W \cap C_{0} \cap V=\emptyset$. Then we consider the disjoint union $C \cup C_{L}$ and the Riemannian surface

$$
\tilde{C}:=C \cup C_{L} / \operatorname{id}_{C_{0}} .
$$

Let $\tilde{f}: U \cup L \rightarrow \tilde{C}$ be induced by $f$ and $f_{L}$. It belongs to $\mathcal{I}$; a contradiction.

2nd case: There exist a point $\sigma \in S$ and a sequence $x_{\nu}$ in $C_{0}$ such that $x_{\nu} \rightarrow \sigma$ in $C$ and $x_{\nu} \rightarrow L$ in $C_{L}$. Applying 1.1 we get an element $\tilde{f}: U \cup L \rightarrow C$ of $\mathcal{I}$; a contradiction.

Theorem 0.3 is proven in the regular case. We got a m.s. integral.

Now let $\operatorname{sing} \mathcal{F} \neq \emptyset$. There exists a m.s. integral $f^{*}: X^{*} \rightarrow C$ of $\mathcal{F}^{*}$ on $X^{*}$. Because of condition (d) we can apply 1.2 and get an integral $f: X \rightarrow C$ of $\mathcal{F}$. It is a m.s. integral.

Proof of 0.5 . We set $\Lambda=X^{*} / \mathcal{F}^{*}, S=\operatorname{sing} \mathcal{F}$. Let $L \in \Lambda$. Then $L$ is a closed subset of $X^{*}$ and therefore an analytic subset of $X^{*}$. By Thullen's extension we conclude that the closure $\bar{L}$ of $L$ is an analytic subset of $X$ because of $\operatorname{dim}$ $S \leq n-2$. We call $L$ singular if $\bar{L} \cap S \neq \emptyset$, otherwise we call it regular. The set of all singular leaves we denote by $\Gamma$.

If $L \in \Gamma$ then there exists an irreducible component $S^{\prime}$ of $S$ such that $S^{\prime} \subset \bar{L}$. If $S^{\prime}$ is an irreducible component of $S$ then the number of leaves $L \in \Gamma$ such that $S^{\prime} \subset \bar{L}$ is greater than zero, but finite.

We need these considerations for proving 0.2 and 0.5 . Now we start with the proof of 0.5 . Let $X / \mathcal{F}$ be Hausdorff. We show that $\Gamma$ satisfies the conditions of 0.3 . (a), (c) and (d) are trivial. Because the sets $\bar{L}, L \in \Gamma$, constitute the irreducible components of sh $\mathcal{F}$, also (b) is valid.

Let $f: X \rightarrow C$ be a m.s. integral of $\mathcal{F}$. Then we can identify $C=(\widetilde{X / \mathcal{F}})=$ $X / \mathcal{F}$.

Proof of 0.2 . We use the same procedure as in the proof of 0.5 and show that $\Gamma$ satisfies the conditions of 0.3 . (a) and $(d)$ are trivial.

Because $S$ is compact it only has a finite number of irreducible components. Therefore $\Gamma$ is finite and (b) is true.

We prove (c). Let be $X_{0}:=\bigcup_{L \in \Lambda \backslash \Gamma} L . X_{0}$ is an open $\mathcal{F}^{*}$-saturated connected subset of $X^{*}$ and $\mathcal{F}$ induces a 1-codimensional regular foliation on $X_{0}$ with compact leaves. Therefore every $L \in \Lambda \backslash \Gamma$ is stable, i.e. every leaf $L \in \Lambda \backslash \Gamma$ has a fundamental system of open $\mathcal{F}$-saturated neighborhoods (comp. [Ka], [Ho 2]). Now consider $L, M \in \Lambda, L \notin \Gamma$. If $M \notin \Gamma$ then there exist disjoint open neighborhoods of $L$ resp. $M$ in $\Lambda$. Let be $M \in \Gamma$ and assume that there are no disjoint open neighborhoods of $L$ resp. $M$ in $\Lambda$. 
Consider a distance $d$ on $X$ defining the topology of $X$ and consider the space $\mathcal{A}(X):=\{Y \subset X: Y$ non-empty and closed $\}$. We equip $\mathcal{A}(X)$ with the Hausdorff distance $\delta$. Then $(\mathcal{A}(X), \delta)$ is a compact metric space.

By our assumption we get a sequence $L_{\nu} \in \Lambda \backslash \Gamma$ converging in $\mathcal{A}(X)$ such that $L \cup M \subset \lim L_{\nu}$. Because $L$ is stable we conclude that $\lim L_{\nu}=L, L=M$; a contradiction.

The conditions of 0.3 are satisfied.

Let $f: X \rightarrow C$ be a m.s. integral of $\mathcal{F}$. We show that $f$ is simple. $f$ is constant on the leaves of $\mathcal{F}$. We show that $f$ separates different leaves of $\mathcal{F}$. We argue indirectly. Let $L, M \in \Gamma$. By $\tilde{L}, \tilde{M}$ we denote the leaves of $\mathcal{F}$ defined by $L$ resp. $M$. Assume that $\tilde{L} \neq \tilde{M}$, but $f(\tilde{L})=f(\tilde{M})$. Because $f$ is open there exists a converging sequence $L_{\nu} \in \Lambda \backslash \Gamma$ such that $L \cup M \subset \lim L_{\nu}$. Because $\lim L_{\nu}$ is connected and $\tilde{L} \neq \tilde{M}$ we can find a point $a \in X_{0} \cap \lim L_{\nu}$. Let $N$ be the leaf passing through $a$. Then we get $\lim L_{\nu}=N, L=M=N$; a contradiction.

3. Remarks. For the following we refer to [Rf 3]. A more official publication will be made by G. Bohnhorst.

Again we consider a connected paracompact complex manifold of dimension $n$ and a holomorphic foliation $\mathcal{F}$ on $X$ of codimension 1.

Using the convergence techniques of the proof for 0.2 and using a local stability theorem of Bohnhorst one can give a new geometrical proof for theorem M/M.

In a similar way and using an idea of Milnor ([Mi]) and techniques of semianalytic geometry ([Ło]) one can prove a semiglobal generalization of theorem M/M (theorem 0.2 in $[\operatorname{Rf} 3])$ :

3.1. TheOREM. Assume that $\mathcal{F}$ is geometrically simple and let $K$ be a compact subset of a leaf of $\mathcal{F}$. Then there exist an open neighborhood $U$ of $K$ in $X$ and an integral $f$ of $\mathcal{F}$ on $U$.

In 3.1 we can choose $U$ connected and $f$ simple. Then $U /(\mathcal{F} \mid U)$ is a Riemannian surface.

Modifying the proof of 0.2 a little bit one can show (corollary 0.5 in [Rf 3]):

3.2. TheOREM. Let $\mathcal{F}$ be geometrically simple and $\operatorname{sh} \mathcal{F}$ an analytic subset of $X$. Assume that every regular leaf of $\mathcal{F}^{*}$ is compact. Then $X / \mathcal{F}$ is a Riemannian surface.

Applying theorem 3.1 and 3.2 we get the following result (corollary 0.6 in $[\operatorname{Rf} 3])$ :

3.3. Theorem. Let $\mathcal{F}$ be geometrically simple. Assume that all leaves of $\mathcal{F}$ are compact. Then $X / \mathcal{F}$ is a Riemannian surface.

This is a generalization of Satz 3 in [Ka] resp. proposition 6.2 in [Ho 2] and of course of our corollary 0.4 . 


\section{References}

[B/R] G. Bohnhorst und H.-J. Reiffen, Holomorphe Blätterungen mit Singularitäten, Math. Gottingensis, Schriftenreihe des Sonderforschungsbereichs Geometrie und Analysis, Heft 5, 1985.

[Fo] O. Forster, Riemannsche Flächen, Heidelberger Taschenbücher 184, Springer, Berlin, 1977.

[Ho 1] H. Holmann, Holomorphe Blätterungen komplexer Räume, Comment. Math. Helv. 47 (1972), 185-204.

[Ho 2] -, On the stability of holomorphic foliations with all leaves compact, in: Variétés analytiques compactes, Colloque Nice, 1977, Lecture Notes in Math. 683, Springer, 1978.

[Hu] H. Huber, Über analytische Abbildungen Riemannscher Flächen in sich, Comm. Math. Helv. 27 (1953), 1-73.

[Ka] B. Kaup, Ein geometrisches Endlichkeitskriterium für Untergruppen von Aut $(\mathbb{C}, 0)$ und holomorphe 1-codimensionale Blätterungen, Comment. Math. Helv. 53 (1978), 295-299.

[Ko] Sh. Kobayashi, Hyperbolic Manifolds and Holomorphic Mappings, Pure and Appl. Math. 2, Marcel Dekker, New York, 1970.

[Kw] M. H. Kwack, Generalization of the big Picard Theorem, Ann. of Math. 90 (1969), $9-22$.

[Ło] S. Łojasiewicz, Ensembles semianalytiques, Institut des Hautes Etudes Scientifiques, Bures-sur-Yvette, France, 1965.

$[\mathrm{M} / \mathrm{M}]$ J. F. Mattei et R. Moussu, Holonomie et intégrales premières, Ann. Sci. École Norm. Sup. (4) 13 (1980), 469-523.

[Mi] J. Milnor, Singular Points of Complex Hypersurfaces, Ann. of Math. Stud. 61, Princeton University Press and the University of Tokyo Press, 1968.

[Rf 1] H.-J. Reiffen, Einfache holomorphe Funktionen, Math. Ann. 259 (1982), 99-106.

[Rf 2] -, Leaf spaces and integrability, in: Holomorphic Dynamics, Proceedings of the Second International Colloquium on Dynamical Systems, Mexico, 1986, Lecture Notes in Math 1345, Springer, 1988.

[Rf 3] - Integrals for holomorphic foliations of codimension one, Osnabrücker Schriften zur Mathematik, Reihe M, Heft 11, 1992. 